
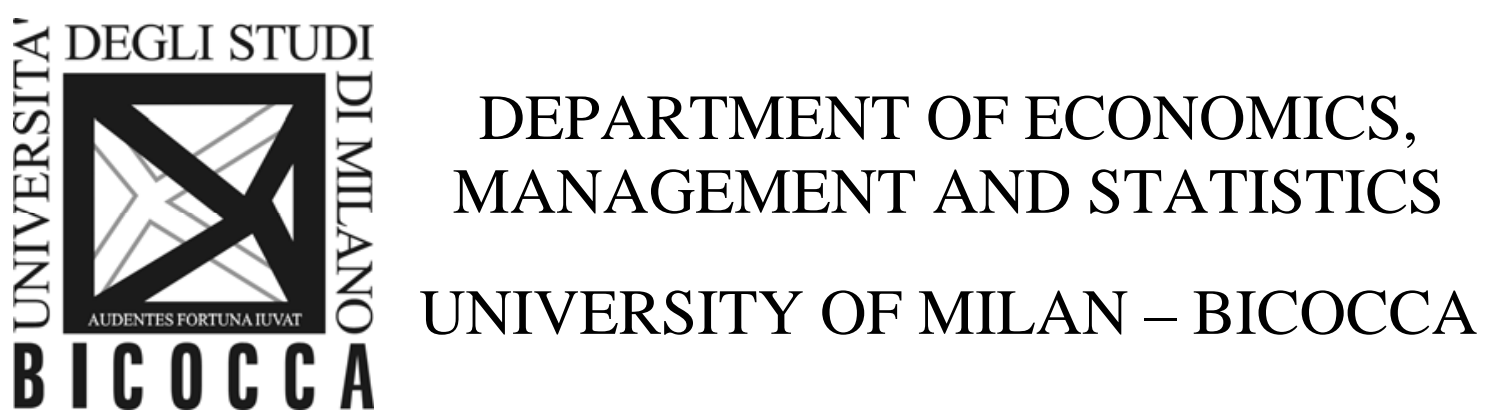

DEMS WORKING PAPER SERIES

\title{
Oil Price Forecastability and Economic Uncertainty
}

Stelios Bekiros, Rangan Gupta, Alessia Paccagnini

No. 298 - April 2015

Dipartimento di Economia, Metodi Quantitativi e Strategie di Impresa Università degli Studi di Milano - Bicocca

http://dems.unimib.it/ 


\title{
Oil Price Forecastability and Economic Uncertainty
}

\author{
Stelios Bekiros ${ }^{\mathrm{a}, \mathrm{b} 1} \quad$ Rangan Gupta $^{\mathrm{a}, \mathrm{c} 2} \quad$ Alessia Paccagnini $^{\mathrm{d} 3}$
}

${ }^{\mathrm{a}}$ IPAG Business School,${ }^{\mathrm{b}}$ European University Institute,${ }^{\mathrm{c}}$ University of Pretoria, ${ }^{\mathrm{d}}$ Università degli Studi di Milano - Bicocca

${ }^{1 a}$ IPAG Lab, IPAG Business School, 184 Boulevard Saint-Germain, 75006 Paris, France.; Tel.: +33015363 3600 ; Fax: +33 01454440 46; ${ }^{b}$ European University Institute, Department of Economics, Via della Piazzuola; 43, I-50133, Florence, Italy; Tel.: +39 0554685 916; Fax: +39 0554685 902; E-mail address: stelios.bekiros@eui.eu;

${ }^{2}$ Department of Economics, University of Pretoria, Pretoria, 0002, South Africa. E-mail address: rangan.gupta@up.ac.za

${ }^{3}$ Corresponding author: Department of Economics, Università degli Studi di Milano - Bicocca - Milan 20126 Tel +39 026448 3046, Email: alessia.paccagnini@unimib.it. 


\begin{abstract}
Information on economic policy uncertainty (EPU) does matter in predicting oil returns especially when accounting for omitted nonlinearities in the relationship between these two variables via a time-varying coefficient approach. In this work, we compare the forecastability of standard, Bayesian and TVP-VAR models against the random-walk and benchmark AR models. Our results indicate that over the period 1900:1-2014:2 the time-varying VAR model with stochastic volatility outranks all alternative models.
\end{abstract}

JEL Classification: C22, C32, C53, E60, Q41

Keywords: Oil prices, economic policy uncertainty, forecasting 


\section{Introduction}

Hamilton (2008) indicates that nine out of ten recessions in the US since World War II have been preceded by an increase in oil prices. Interestingly, Hamilton (2009) goes as far as arguing that a large proportion of the recent downturn in the US GDP during the 'Great Recession' can be attributed to the oil price shock of 2007-2008. Stock and Watson (2003) also show the ability of oil price in predicting growth and inflation. A recent strand of literature emphasizes the role of economic policy uncertainty (EPU) on real activity (Bloom, 2009; Jones and Olson, 2013), which in turn affects oil-price movements, as depicted in Kang and Ratti (2013a, b) and Antonakakis et al. (2014). To the best of our knowledge, this is the first attempt to forecast oil returns using a news-based measure of EPU. This measure, developed by Baker et al., (2013), relies on an automated text-search process of large US newspapers and identifies articles that use words related to economic policy, regulation and uncertainty. In our approach we compare the ability of VAR, standard Bayesian VARs and time-varying parameter VARs, against random-walk and univariate AR models of real oil returns over the monthly out-of-sample period 2007:1-2014:2, using an extended in-sample period of 1900:1-2006:12. The paper is organized as follows: section 2 briefly presents the various econometric models and section 3 discusses the data and results; finally section 4 concludes.

\section{Econometric Models}

The econometric models used include the classical and Bayesian VAR, a time-varying VAR with stochastic volatility (TVP-VAR) and a new TVP-VAR with Markov-switching heteroscedasticity as in Bekiros and Paccagnini (2014). Our benchmark models are the random-walk (RW) and an $\operatorname{AR}(p)$ model.

\subsection{Classical VAR}

As suggested by Sims (1980) it has the following compact format 


$$
\mathbf{Y}_{t}=\mathbf{X}_{t} \mathbf{\Phi}+\mathbf{U}
$$

where $\mathbf{Y}_{t}$ is a $(T \times n)$ matrix with rows $Y_{t}^{\prime}, \mathbf{X}$ is a $(T \times k)$ matrix $(k=1+n p, p=$ number of lags) with rows $X_{t}^{\prime}=\left[1, Y_{t-1}^{\prime}, \ldots, Y_{t-p}^{\prime}\right] . \quad \mathbf{U}$ is a $(T \times n)$ matrix with rows $u_{t}^{\prime}, \boldsymbol{\Phi}$ is a $(k \times n)=\left[\Phi_{0}, \Phi_{1}, \ldots, \Phi_{p}\right]^{\prime}$, while the one-step ahead forecast errors $u_{t}$ have a multivariate $N\left(0, \boldsymbol{\Sigma}_{u}\right)$ conditional on past observations of $\mathbf{Y}$. Based on the Akaike information criterion the optimal lag $p$ is set at 6 .

\subsection{BVAR model}

A Bayesian VAR (BVAR) imposes restrictions on the numerous VAR parameters by specifying normal prior distributions with zero means and small standard deviations for all coefficients, with a decreasing standard deviation as the lags increase. Popular priors are the "Minnesota " ones, written as follows

$$
\Phi_{i} \sim N\left(1, \sigma_{\Phi_{i}}^{2}\right) \text { and } \Phi_{j} \sim N\left(0, \sigma_{\Phi_{j}}^{2}\right)
$$

where $\Phi_{i}$ denotes the coefficients associated with the lagged dependent variables in each equation of the VAR, while $\Phi_{j}$ represents any other coefficient. The prior variances $\sigma_{\Phi_{i}}^{2}$ and $\sigma_{\Phi_{j}}^{2}$ specify the uncertainty of the prior means, $\Phi_{i}=1$ and $\Phi_{j}=0$, respectively. In this study, since our variables are mean-reverting, we impose $\Phi_{i}=0$ as well, i.e., a white-noise mean prior. The specification of the standard deviation of the distribution of the prior imposed on variable $j$ in equation $i$ at lag $m$, for all $i, j$ and $m$, denoted by $S(i, j, m)$, is specified as follows

$$
S(i, j, m)=[w \times g(m) \times F(i, j)] \frac{\hat{\sigma}_{i}}{\hat{\sigma}_{j}}
$$

where

$$
F(i, j)=\left\{\begin{array}{c}
1 \text { if } i=j \\
k_{i j} \text { otherwise, } 0 \leq k_{i j} \leq 1
\end{array}\right.
$$


is the tightness of variable $j$ in equation $i$ relative to variable $i$ and by increasing the interaction, i.e. it is possible for the value of $k_{i j}$ to loosen the prior. The term $w$ measures the standard deviation on the first lag, and also indicates the overall tightness. The function $g(m)=m^{-d}, d>0$ is the measurement of the tightness on lag $m$ relative to lag 1 , and is assumed to have a harmonic shape with a decay of $d$, which tightens the prior on increasing lags. Following the literature on the Minnesota prior settings, we experimented with various combinations $(0.3,0.5 ; 0.1,1.0 ; 0.2,1.0 ; 0.2,2.0 ; 0.1,2.0)$ of $w$ and $d$ respectively, with $k_{i j}$ set equal to 0.5 . We found that $w=0.3$ and $d=0.5$ produced the best out-of-sample forecast on average, and hence, we only report the results based on this prior setting for the BVAR.

\section{$2.3 \quad$ TVP-VAR model}

As evidenced in D'Agostino et al. (2013), a VAR with time-varying parameters and stochastic volatility can predict well many US macroeconomic variables. To model oil price and uncertainty, we implement the following set up:

$$
\mathbf{Y}_{t}=c_{t}+\sum_{j=1}^{K} \mathbf{B}_{t} \mathbf{Y}_{t-j}+\Omega_{t}^{1 / 2} \varepsilon_{t}
$$

where the VAR coefficients $\Phi_{t}=\operatorname{vec}\left(\left\{c_{t}, B_{t}\right\}\right)$ evolve as random walks, $\Phi_{t}=\Phi_{t-1}+\eta_{t}$. Following Cogley and Sargent (2005), the covariance matrix of the innovations $v_{t}\left(\Omega_{t}\right)$ can be factored as:

$$
\mathbf{\Omega}_{t}=\mathbf{A}_{t}^{-1} \mathbf{H}_{t}\left(A_{t}^{-1}\right)^{\prime \prime}
$$

where 


$$
\begin{aligned}
& \mathbf{A}_{t}=\left[\begin{array}{cc}
1 & 0 \\
\alpha_{21, t} & 1
\end{array}\right] \text { and } \\
& \mathbf{H}_{t}=\left[\begin{array}{cc}
h_{1, t} & 0 \\
0 & h_{2, t}
\end{array}\right]
\end{aligned}
$$

with $h_{i, t}$ evolving as geometric random walks: $\ln h_{i, t}=\ln h_{i, t-1}+\widetilde{\nu}_{t}$. We assume that the non-zero and non-one elements of the matrix $A_{t}$ is a RW such as $\alpha_{21, t}=\alpha_{21, t-1}+\tau_{t}$. The model is estimated allowing for stochastic volatility in $\eta_{t}$, as suggested in Baumeister et al. (2013) and Cogley et al. (2008). In this last case, Barnett et al. (2014) evidence how the VAR coefficients may change faster during the recent crisis, while the changes are small in tranquil periods, hence the TVP-VAR is a better forecasting tool during turmoil events. We estimate the model using a Gibbs sampling algorithm and the posterior simulated is computed as proposed by Carter and Kohn (2004).

\subsection{TVP-VAR model with Markov-Switching Heteroscedasticity}

We utilize the multivariate state-space TVP-VAR model of Bekiros and Paccagnini (2014) with $h_{t}$ the error of the measurement equation and $Q_{t}$ the covariance matrix of the state equation. Both equations incorporate time varying coefficient and state transition matrices. As an alternative to the classical TVP-VAR with homoskedastic volatility (stochastic or not) they assume that the measurement and state equation error structure is dependent on unobserved discrete-time, discrete-state Markov processes (TVP-VAR-MS). To estimate the model they introduce a Quasi-optimal Kalman filtering approach with two-state Markovswitching heteroskedasticity. Their set-up is intended to capture a low- and high-volatility regime, as observed in prior and post-crisis periods. They consider the following first-order, $\omega$-state Markov-switching model of heteroskedasticity: 


$$
\begin{array}{r}
Q_{t}=Q^{S_{t}}=Q_{1} \Theta_{1 t}+Q_{2} \Theta_{2 t}+\cdots+Q_{\omega} \Theta_{\omega t} \\
h_{t}=h^{S_{t}}=h_{1} \Theta_{1 t}+h_{2} \Theta_{2 t}+\cdots+h_{\omega} \Theta_{\omega t} \\
p=\left(\begin{array}{cccc}
p_{11} & p_{12} & \cdots & p_{1 \omega} \\
p_{21} & p_{22} & \cdots & p_{2 \omega} \\
\vdots & \vdots & \ddots & \vdots \\
p_{\omega 1} & p_{\omega 2} & \cdots & p_{\omega \omega}
\end{array}\right)
\end{array}
$$

where $\Theta_{j t}=1$ if $S_{t}=j$ and $\Theta_{j t}=0$ if $S_{t} \neq j(j=1,2, \ldots, \omega), p_{i j}=\operatorname{Pr}\left[S_{t}=j \mid S_{t-1}=i\right]$, for $i, j=1,2, \ldots, \omega$, and $\sum_{j=1}^{\omega} p_{i j}=1$. The unobserved-state variable $S_{t}$ evolves according to a Markov process with the transition probability matrix. In particular for a two-state Markovswitching model of heteroskedasticity: $\operatorname{Pr}\left[S_{t}=1 \mid S_{t-1}=1\right]=P_{11} ; \operatorname{Pr}\left[S_{t}=0 \mid S_{t-1}=1\right]=$ $1-P_{11} ; \operatorname{Pr}\left[S_{t}=1 \mid S_{t-1}=0\right]=1-P_{00} ; \operatorname{Pr}\left[S_{t}=0 \mid S_{t-1}=0\right]=P_{00}$. They estimate the hyperparameters via an approximated conditional log-likelihood function and show how prediction and smoothing can be obtained. The decoupling of the Kalman filter is derived as

$$
\mathbf{y}_{t}=\left(\mathbf{z}_{t}^{\prime} \otimes \mathbf{I}_{N}\right) \boldsymbol{\alpha}_{t}+\boldsymbol{\varepsilon}_{t}, \text { with } \operatorname{Var}\left(\varepsilon_{t}\right)=\operatorname{Var}\left(h_{t}^{j}\right) \boldsymbol{\Sigma}_{*}=\boldsymbol{\Sigma}_{h^{j}} \boldsymbol{\Sigma}_{*}
$$

$$
\boldsymbol{\alpha}_{t}=\left(\mathbf{T}_{t} \otimes \mathbf{I}_{N}\right) \boldsymbol{\alpha}_{t-1}+\left(\mathbf{R}_{t} \otimes \mathbf{I}_{N}\right) \boldsymbol{\eta}_{t}, \text { with } \operatorname{Var}\left(\boldsymbol{\eta}_{t}\right)=\operatorname{Var}\left(Q_{t}^{j}\right) \otimes \boldsymbol{\Sigma}_{*}=\boldsymbol{\Sigma}_{Q^{j}} \otimes \boldsymbol{\Sigma}_{*}
$$

where $\operatorname{Var}\left(\varepsilon_{t}\right)=\operatorname{Var}\left(h_{t}^{j}\right)=\boldsymbol{\Sigma}_{h^{j}}$ and $\operatorname{Var}\left(\boldsymbol{\eta}_{t}\right)=\operatorname{Var}\left(Q_{t}^{j}\right)=\boldsymbol{\Sigma}_{Q^{j}}$ are block diagonal matrices, although a more general formulation does not constrain them to be diagonal. However, restrictions are needed on the matrices for the model to be identifiable. The technical specificities of the model can be found in Bekiros and Paccagnini (2014). Finally, prediction is produced via $\mathbf{P}_{t+1 \mid t}^{(i, j) *}=\mathbf{P}_{t+1 \mid t}^{(i, j)} \otimes \mathbf{\Sigma}_{*}$, where $\mathbf{P}_{t+1 \mid t}^{(i, j)}$ is the MSE matrix from the corresponding univariate model. 


\section{Data and Results}

The two variables of concern comprise real oil prices obtained by dividing the Western Texas Intermediate (WTI) by the Consumer Price Index (CPI), and EPU. Standard unit root tests indicate that the log-EPU is stationary as opposed to the logarithm of real oil price, hence the latter is transformed to real oil returns (month-on-month growth rate of real oil price) to ensure mean-reversion. In order to further rationalize the use of TVPVARs we employed the BDS test (Brock et al., 1996) which overwhelmingly rejected the null hypothesis that AR- or VAR-filtered errors are i.i.d for all possible dimensions, thus implying an omitted nonlinear structure. ${ }^{1}$

\begin{tabular}{ccccccc}
\multicolumn{7}{c}{ Table 1: Root Mean Square Errors of Real Oil Returns $(2007: 1-2014: 2)$} \\
\hline \hline$h$ & RW & AR & VAR & BVAR & TVP-VAR & TVP-VAR- \\
& & & & & & MS \\
\hline 1 & 10.1183 & 0.8490 & 0.8437 & 0.8428 & $0.2476^{*}\left(^{*}\right)$ & 0.8982 \\
2 & 10.1960 & 0.8361 & 0.8336 & 0.8340 & $0.2095^{*}\left(^{*}\right)$ & 0.8378 \\
3 & 12.3897 & 0.7013 & 0.7001 & 0.7005 & $0.5662^{*}\left(^{*}\right)$ & 0.7250 \\
4 & 12.9333 & 0.6746 & 0.6730 & 0.6735 & $0.5467^{*}\left(^{*}\right)$ & 0.7100 \\
5 & 13.6891 & 0.6398 & 0.6375 & 0.6381 & $0.4650^{*}\left(^{*}\right)$ & 0.6517 \\
6 & 14.7744 & 0.5961 & 0.5921 & 0.5930 & $0.4795^{*}\left(^{*}\right)$ & 0.5932 \\
7 & 14.1876 & 0.6326 & 0.6306 & 0.6311 & $0.5277^{*}\left(^{*}\right)$ & 0.6248 \\
8 & 13.8192 & 0.6518 & 0.6512 & 0.6514 & $0.5083^{*}\left(^{*}\right)$ & 0.6422 \\
9 & 13.4794 & 0.6729 & 0.6733 & 0.6732 & $0.4913^{*}\left(^{*}\right)$ & 0.6507 \\
10 & 13.3569 & 0.6784 & 0.6786 & 0.6785 & $0.4721^{*}\left(^{*}\right)$ & 0.6673 \\
11 & 13.4487 & 0.6756 & 0.6757 & 0.6756 & $0.4504^{*}\left(^{*}\right)$ & 0.6600 \\
12 & 12.8896 & 0.7040 & 0.7039 & 0.7039 & $0.4714^{*}\left({ }^{*}\right)$ & 0.6938 \\
13 & 13.7027 & 0.6665 & 0.6664 & 0.6664 & $0.6174^{*}$ & 0.6450 \\
14 & 14.0264 & 0.6555 & 0.6555 & 0.6555 & $0.5882^{*}\left({ }^{*}\right)$ & 0.6325 \\
15 & 14.0290 & 0.6595 & 0.6592 & 0.6593 & $0.6200^{*}$ & 0.6316 \\
16 & 13.5331 & 0.6837 & 0.6834 & 0.6834 & $0.6600^{*}$ & 0.6785 \\
17 & 13.1287 & 0.7077 & 0.7074 & 0.7074 & $0.6672^{*}$ & 0.6731 \\
18 & 13.3066 & 0.6976 & 0.6973 & 0.6974 & $0.6497^{*}$ & 0.6722 \\
19 & 12.7567 & 0.7311 & 0.7308 & 0.7308 & $0.6987^{*}$ & 0.6980 \\
20 & 13.6419 & 0.6887 & 0.6886 & 0.6886 & $0.6373^{*}$ & 0.6602 \\
21 & 12.4713 & 0.7481 & 0.7478 & 0.7479 & $0.6855^{*}$ & 0.7177 \\
22 & 13.3449 & 0.6967 & 0.6967 & 0.6967 & $0.6383^{*}$ & 0.6854 \\
23 & 12.6627 & 0.6794 & 0.6794 & 0.6794 & $0.6954^{*}$ & 0.7314 \\
24 & 13.6014 & 0.5859 & 0.5859 & 0.5860 & $0.6866^{*}$ & 0.6851 \\
\hline \hline Average & & 0.6880 & 0.6872 & 0.6873 & 0.5533 & 0.6861 \\
\hline \hline
\end{tabular}

Notes: Entries for the RW model are the RMSEs in percentage, while rest of the entries are the relative RMSEs with respect to the RW; ${ }^{*}$ ( or ${ }^{\#}$ ) indicates significance of the MSE - F statistic with respect to the RW (AR) model at $1 \%(1 \%$ or $5 \%)$ level of significance.

We analyze the ability of the EPU to forecast real oil returns over the period 2007:12014:2, i.e., during the recent global crisis, using an in-sample period spanning 1900:12006:12. All the models are estimated recursively over the out-of-sample period to produce 1- to 24-months-ahead RMSEs. In Table 1, we report the RMSEs from the RW whilst the rest of the models are reported relative to the RW. Based on the results, we can draw the

\footnotetext{
${ }^{1}$ Details are available upon request from the authors.
} 
following conclusions: (a) all models outperform the RW at all horizons; (b) on average, the TVP-VAR model with stochastic volatility is the best performer followed by the TVPVAR-MS; (c) in specific horizons, the TVP-VAR produces the maximum gain at 1- to 18and 20- to 22-steps-ahead, while the TVP-VAR-MS is the best for the 19-month-ahead and the AR model at 23- and 24-steps-ahead; (d) finally, using the $M S E-F$ test statistic of McCracken (2007) we confirm that the TVP-VAR model statistically outperforms the RW at $1 \%$ significance level at all horizons ${ }^{2}$ and the AR model at horizons of 1 - to 12 -stepsahead (1\% level) and 14-months (5\%). Overall, information on EPU helps in forecasting real oil returns when allowing for adaptive learning or inherent nonlinearities as captured by the TVP-VARs ${ }^{3}$

\section{Conclusions}

The importance of oil prices in determining movements of US growth and inflation is wellestablished, hence accurate forecasting is of paramount importance. Moreover, recent works in the literature advocate in favor of economic policy uncertainty driving oil-price fluctuations. Against this backdrop, we compare the forecastability of various uni- and multivariate models of real oil returns and EPU. Our results indicate that TVP-VARs outperform the others in all horizons till two-years-ahead relative to the benchmark Random Walk. Consequently, information on EPU does matter in predicting oil returns out-of-sample, especially when accounting for adaptive nonlinearities in the relationship between these two variables via a time-varying coefficient approach.

\footnotetext{
${ }^{2}$ This test compares the null of equal forecasting ability between a restricted (RW or AR) and an unrestricted model (in our case the best performing VAR on average, i.e., TVP-VAR). For all the other models, the $M S E-F$ is significant at least at the $5 \%$ level over all horizons. Complete details are available upon request from the authors.

${ }^{3}$ The TVP-VAR-MS allows for regime shifts, yet the particular out-of-sample period does not incorporate various regimes but only one i.e., the crisis period. Qualitatively similar results were obtained when we used other periods based on identified structural breaks by the Bai and Perron (2003) tests e.g., 1986:9 or 1999:1, the latter corresponding to the introduction of the euro.
} 


\section{References}

Antonakakis, N., Chatziantoniou, I. \& Filis, G., (2014). Dynamic spillovers of oil price shocks and economic policy uncertainty. Energy Economics 44, 433-447.

Bai, J. \& Perron, P. (2003). Computation and analysis of multiple structural change models, Journal of Applied Econometrics, 18, 1-22.

Baker, Scott R. and Bloom, Nicholas \& Davis, Steven J., (2013). Measuring Economic Policy Uncertainty. Chicago Booth Research Paper No. 13-02.

Barnett, A., Mumtaz, H., Theodoridis, K. (2014). Forecasting UK GDP growth and inflation under structural change. A comparison of models with time-varying parameters. International Journal of Forecasting, Volume 30(1), 129-143.

Baumeister, C., Liu, P., \& Mumtaz, H. (2013). Changes in the effects of monetary policy on disaggregate price dynamics. Journal of Economic Dynamics and Control, 37, 543-560.

Bekiros, S. and Paccagnini, A. (2014). Macroprudential policy and forecasting using Hybrid DSGE models with financial frictions and State space Markov-Switching TVP-VARs. Macroeconomic Dynamics, doi: 10.1017/S1365100513000953

Bloom, N., (2009). The impact of uncertainty shocks. Econometrica 77, 623-685.

Brock, W., Dechert, D., Scheinkman, J. \& LeBaron, B. 1996. A Test for Independence Based on the Correlation Dimension. Econometric Reviews, 15, 197-235.

Carter, C. K., \& Kohn, R. (2004) On Gibbs Sampling for State Space Models. Biometrika, 81: 541-553.

Cogley, T., Primiceri, G.E., \& Sargent, T.J. (2008). Inflation-gap persistence in the US. NBER working paper: vol. 13749. National Bureau of Economic Research, Inc.

Cogley, T., \& Sargent, T. J. (2005). Drifts and volatilities: monetary policies and outcomes in the post WWII US. Review of Economic Dynamics, 8, 262-302. 
D’Agostino, A., Gambetti, L., \& Giannone, D. (2013). Macroeconomic forecasting and structural change. Journal of Applied Econometrics, 28, 82-101.

Hamilton, J. D., (2008). Oil and the Macroeconomy, in New Palgrave Dictionary of Economics: Eds. S.Durlauf and L. Blume, Palgrave McMillan Ltd.

Hamilton, J. D., (2009). Causes and Consequences of the Oil Shock of 2007-08, Brookings Papers on Economic Activity, Spring: 215-259.

Jones, P.M. and (2013). The time-varying correlation between uncertainty, output, and inflation: Evidence from a DCC-GARCH model. Economics Letters, 118, 33-37.

Kang, W. \& Ratti, R. A. (2013a). Structural oil price shocks and policy uncertainty. Economic Modelling 35, 314-319.

Kang, W. \& Ratti, R.A., (2013b). Oil shocks, policy uncertainty and stock market return. International Financial Markets. Institutions and Money 26, 305-318.

McCracken, M.W., (2007). Asymptotics for out-of-sample tests of Granger causality, Journal of Econometrics, 140, 719-752.

Stock, J.H. \& Watson, M.W. (2003). Forecasting Output and Inflation: The Role of Asset Prices. Journal of Economic Literature 41, 788-829. 